\title{
Treatment of iron deficiency and iron deficiency anemia with intravenous ferric carboxymaltose in pregnancy
}

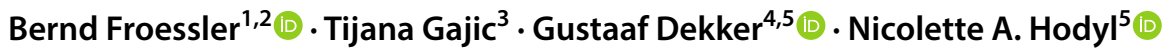

Received: 30 January 2018 / Accepted: 30 April 2018 / Published online: 8 May 2018

(c) The Author(s) 2018

\begin{abstract}
Purpose To evaluate the efficacy and safety of intravenous ferric carboxymaltose administration to pregnant women with varying severities of iron deficiency anemia and iron deficiency without anemia.

Methods In this prospective observational study of local obstetric practice, we analyzed data from 863 pregnant women with iron deficiency according to anemia status and severity. All women were treated with intravenous ferric carboxymaltose in pregnancy. Treatment efficacy was assessed by repeat hemoglobin measurements at 3 and 6 week post-infusion and ferritin levels, where available. Safety was assessed by analysis of adverse events, fetal heart rate monitoring, and newborn health outcome data. Results Ferric carboxymaltose significantly increased hemoglobin in women with mild, moderate, and severe iron deficiency anemia and women with iron deficiency alone at 3 and 6 week post-infusion $(p<0.01$ for all). No hemoconcentration occurred in iron-deficient women without anemia. No serious adverse events were recorded, with minor temporary side effects (including local skin irritation, nausea, and headache) occurring in 96 (11\%) women. No adverse fetal or neonatal outcomes were observed.

Conclusions Ferric carboxymaltose infusion corrects iron deficiency or various degrees of iron deficiency anemia efficaciously and safely pregnant women, and does not cause hemoconcentration.
\end{abstract}

Keywords Pregnancy $\cdot$ Iron deficiency no anemia $\cdot$ Iron deficiency anemia severity $\cdot$ Intravenous iron $\cdot$ Ferric carboxymaltose $\cdot$ Safety

\section{Introduction}

Iron deficiency (ID) and iron deficiency anemia (IDA) in pregnancy are global health issues, affecting around $30 \%$ of women in high-resourced countries, and increasing to over $50 \%$ of women in low-resourced countries [1]. It is well recognized in that both conditions are associated with adverse

Bernd Froessler

bernd.froessler@sa.gov.au

1 Department of Anesthesia, Lyell McEwin Hospital, Haydown Road, Elizabeth Vale, SA 5112, Australia

2 Discipline of Acute Care Medicine, University of Adelaide, Adelaide, SA 5005, Australia

3 Flinders Medical Centre, Bedford Park, SA 5042, Australia

4 Department of Obstetrics and Gynecology, Lyell McEwin Hospital, Elizabeth Vale, SA 5112, Australia

5 The Robinson Research Institute, Adelaide Medical School, University of Adelaide, Adelaide, SA 5006, Australia physiological and psychological outcomes in mother and child. For the mother, these include cardiovascular problems, reduced physical activity and cognitive performance, reduced immune function, tiredness, and increased depressive episodes, while, for the infant, these include preterm birth, fetal growth restriction, intrauterine fetal death, low Apgar scores, and neonatal infection [2-6].

Uncertainty remains whether non-anemic iron-deficient pregnant women require iron replacement and whether intravenous (IV) iron should be considered or could potentially be concerning by causing hemoconcentration [5]. In other patient groups, iron-deficient non-anemic (IDNA) individuals were found to have worse mental, physical health outcomes, executive functioning (EF), which were discharged later from hospital after surgery and had higher mortality rates at 90 days after hospital discharge [7-9].

In addition, women entering labor in an anemic state have a reduced ability to compensate for peri-partum hemorrhage and are, therefore, at increased risk for morbidity and mortality [10]. This risk can become amplified in the context of 
caesarean section, given the increased blood loss that occurs with this intervention [11]. Anemia also increases the need for a peri-partum allogeneic red blood cell (RBC) transfusion, which is independently associated with increased morbidity $[11,12]$. While RBC transfusion often remains the default treatment option [13], it only corrects hemoglobin temporarily and not the underlying condition $[14,15]$.

Iron stores are not routinely assessed in the antenatal setting and iron supplementation is often only initiated when anemia is detected. The adequate assessment and treatment of ID and IDA during pregnancy, as part of routine antenatal care, may, therefore, be beneficial for both maternal and newborn health [16]. Different levels of anemia, patients' response, compliance, and tolerability to oral iron require individualized treatment. Oral iron replacement is often considered as the first-line treatment [17]. However, it can be ineffective, cause intolerable adverse events, or may not facilitate urgent rapid iron repletion [18, 19]. Intravenous iron administration is an alternative treatment option for IDA in pregnancy and has been recommended in various guidelines [17]. The uptake of this administration route has been hindered by perceived barriers and misconceptions, discussed elsewhere [20-22], and has led to the development of new, safer types of iron formulation. Data from observational studies on the efficacy and safety of these new treatments are critical to guide clinical management decisions, and to assure the safety of expecting mothers and the unborn fetus. Christoph et al. demonstrated a comparable safety profile to iron sucrose for ferric carboxymaltose (FCM) in a retrospective analysis [23]. Our group also reported the safe and effective use of FCM in the treatment of ID/IDA in the second and third trimesters of pregnancy in a small observational study $(n=65)$ [21] whether women with ID, mild, moderate, and severe anemia tolerate this treatment, and whether it is successful in the correction of a low hemoglobin in women not responding to oral iron or too late in gestation. However, data on FCM administration during pregnancy remain limited and have been published only from six studies in a total of 634 women [17]. In addition, there is a little knowledge about the erythropoietic response to IV iron in pregnant women with IDNA and IV iron administration appears to be a concern for many clinicians [22]. Therefore, we now assess the efficacy and safety of this treatment in the so far largest single cohort, a further 863 pregnant women, according to the baseline severity of ID/IDA, to enhance general knowledge and to examine the effect on pregnant women with IDNA.

\section{Methods}

This retrospective study was approved by the Queen Elizabeth Hospital, Lyell McEwin Hospital, and Modbury Hospital Human Research and Ethics committee (Reference number 2011160). Consent from individual participants was not required, because all the measurements and demographic information were collected as part of routine care. All women were referred for IV iron administration by the obstetric team for a peri-partum iron infusion as part of individual antenatal care. Risks and benefits were discussed as part of routine clinical management. The most recent hemoglobin and in most cases ferritin results from routine antenatal visits, prompted the decision, mostly since these women were too close to term to opt for oral iron, were not responding to oral iron or not compliant with oral iron treatment.

FCM is the institutional IV iron formulation of choice, and as per hospital protocol, women were prescribed up to a maximum of $20 \mathrm{mg}$ of FCM per $\mathrm{kg}$ bodyweight. The vast majority of women received $1000 \mathrm{mg}$ of FCM.

Available data from women who received FCM infusions as outpatients in the Women's Assessment Unit at the Lyell McEwin Hospital (Elizabeth Vale, South Australia) between August 2012 and December 2016 were analyzed.

All blood samples were collected prior to infusion and then again, where clinically indicated, at up to two postinfusion visits (at approximately 3 and 6 weeks). Hemoglobin and ferritin concentrations were determined in the hospital laboratory using sodium lauryl sulphate (SLS) method for $\mathrm{Hb}$ analysis (Sysmex XE2100 analyzer) and direct chemiluminometric sandwich immunoassay (Siemens ADVIA Centaur XP) for ferritin analysis. Women were observed for $60 \mathrm{~min}$ following the infusion, before being discharged home. Medical and pathology data were collated from case notes and electronic laboratory reports, as well as transfusion data linkage reports.

\section{Statistical analysis}

For analysis, women were subdivided based on their hemoglobin concentrations prior to the iron infusion, with severe anemia defined as $<90 \mathrm{~g} / \mathrm{L}$, moderate anemia defined as 90-94 g/L, mild anemia defined as 95-110 g/L, and iron deficiency with no anemia defined as $>110 \mathrm{~g} / \mathrm{L}$. ANOVA or Kruskal-Wallis tests were used to compare demographic data and baseline hemoglobin and ferritin data between the four ID and anemia groups, using Bonferroni post hoc comparisons (or Mann-Whitney $U$ tests applying a Bonferroni correction) where indicated. Changes in hemoglobin over the pre- and post-infusion 
periods were analyzed comparing across the four groups using repeated-measures ANOVA, with time (pre-infusion, 3 and 6 weeks) as the repeated measure. Due to the loss of follow-up data at the 6 week time point and to maximize the power of the analysis, two separate repeatedmeasures ANOVAs were performed-the first comparing between pre-infusion and 3 weeks and the second comparing between 3 and 6 weeks. To adjust for multiple comparisons, an a priori adjustment was made to the critical $p$ value using the Bonferroni method for this analysis Frequency data (including side effect rates) were analyzed using the Chi-squared test. All analyses were conducted using SPSS (v24). $p$ values $<0.05$ were considered to indicate statistical significance.

\section{Results}

The demographic characteristics of the 863 women receiving FCM for iron deficiency or iron deficiency anemia are presented in Table 1 . At the time of the infusion, women were defined as having mild anemia $(n=462 ; 54 \%)$, while $88(10 \%)$ had moderate anemia, $79(9 \%)$ had severe anemia, and 234 (27\%) had iron deficiency with no anemia. Most women in this study were Caucasian, with a mean BMI in the overweight range. Women with ID and severe anemia were significantly younger $(p=0.01)$ and had a significantly lower BMI than women with ID and no anemia $(p<0.01$; Table 1$)$. Most women gave birth by vaginal delivery. Less than one-third of women in each anemia severity group were taking oral iron supplements

Table 1 Demographic and clinical characteristics of women according to anemia status at infusion

\begin{tabular}{|c|c|c|c|c|c|}
\hline & ID no anemia $(n=234)$ & ID mild anemia $(n=462)$ & $\begin{array}{l}\text { ID moderate } \\
\text { anemia }(n=88)\end{array}$ & ID severe anemia $(n=79)$ & $p$ \\
\hline Age (years) & $28 \pm 6$ & $28 \pm 6$ & $26 \pm 6$ & $26 \pm 6^{\mathrm{a}}$ & 0.01 \\
\hline BMI & $29 \pm 8$ & $27 \pm 8$ & $26 \pm 5$ & $25 \pm 5^{\mathrm{a}}$ & $<0.01$ \\
\hline Gravidity & $3(2-4)$ & $3(2-4)$ & $3(2-4)$ & $3(2-5)$ & 0.84 \\
\hline Parity & $1(1-2)$ & $1(1-3)$ & $1(1-2)$ & $1(0-3)$ & 0.54 \\
\hline \multicolumn{6}{|l|}{ Mode of delivery } \\
\hline Vaginal & $120(51 \%)$ & $260(56 \%)$ & $55(62.5 \%)$ & $51(64.5 \%)$ & 0.50 \\
\hline Elective caesarean & $57(24 \%)$ & $92(20 \%)$ & $9(10 \%)$ & $17(21.5 \%)$ & \\
\hline Emergency caesarean & $31(13 \%)$ & $60(13 \%)$ & $11(12.5 \%)$ & $4(5 \%)$ & \\
\hline Instrumental/episiotomy & $20(9 \%)$ & $42(9 \%)$ & $12(14 \%)$ & $4(5 \%)$ & \\
\hline Unknown & $6(3 \%)$ & $8(2 \%)$ & $1(1 \%)$ & $3(4 \%)$ & \\
\hline \multicolumn{6}{|l|}{ Pre-infusion supplements } \\
\hline Oral iron & $30(13 \%)$ & $94(20 \%)$ & $24(27 \%)$ & $26(33 \%)$ & 0.65 \\
\hline $\begin{array}{l}\text { Oral pregnancy formula (contains } \\
\text { iron) }\end{array}$ & $19(8 \%)$ & $42(9 \%)$ & $12(14 \%)$ & $9(11 \%)$ & 0.38 \\
\hline Gestational diabetes & $47(20 \%)$ & $61(13 \%)$ & $9(10 \%)$ & $10(13 \%)$ & 0.29 \\
\hline Blood loss (estimated) (mL) & $350(250-500)$ & $300(200-500)$ & $300(200-500)$ & $300(200-500)$ & 0.48 \\
\hline $\begin{array}{l}\text { Gestational age at intervention } \\
\text { (weeks) }\end{array}$ & $35(32-37)$ & $35(32-37)$ & $35(32-37)$ & $35(30-36)$ & 0.13 \\
\hline
\end{tabular}

Data are presented as mean $\pm \mathrm{SD}$, median (interquartile range), or $n(\%)$

${ }^{\mathrm{a}} p<0.05$ (post hoc comparison) ID severe anemia compared to ID no anemia

Table 2 Haematological characteristics of women according to anemia status at infusion

\begin{tabular}{lllll}
\hline & ID no anemia $(n=234)$ & ID mild anemia $(n=462)$ & $\begin{array}{l}\text { ID moderate anemia } \\
(n=88)\end{array}$ & ID severe anemia $(n=79)$ \\
\hline $\begin{array}{l}\text { Hemoglobin } \\
\text { at booking } \\
(12 \text { weeks })(\mathrm{g} / \mathrm{L})\end{array}$ & $124(118-131)$ & $115(107-123)^{\mathrm{a}}$ & $113(103-122)^{\mathrm{a}}$ & $106(92-119)^{\mathrm{a}}$ \\
$\begin{array}{l}\text { Ferritin at book- } \\
\text { ing }(12 \text { weeks }) \\
(\mu \mathrm{g} / \mathrm{L})\end{array}$ & $21(10-38)$ & $16(8-34)$ & $12(6-24)$ & $8(5-23)$ \\
\hline
\end{tabular}

Data are presented as median (interquartile range)

${ }^{\mathrm{a}} p<0.05$ (post hoc comparison) in each group compared to the ID with no anemia group 


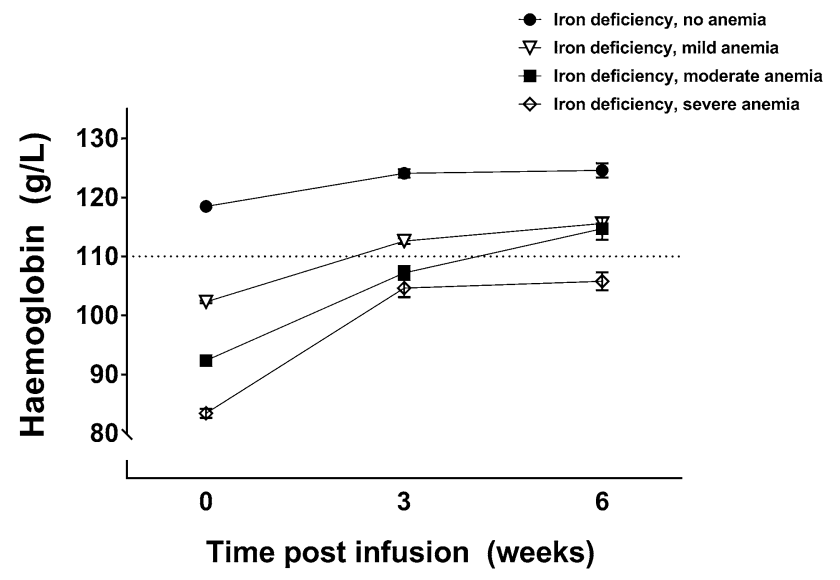

Fig. 1 Hemoglobin levels (mean \pm SEM) across the testing period according to the severity of iron deficiency anemia prior to infusion. Dotted line reflects adequate hemoglobin concentration in second and third trimester of pregnancy $(110 \mathrm{~g} / \mathrm{L})$. No anemia $(\mathrm{n}=234)$, mild anemia $96-110 \mathrm{~g} / \mathrm{L}(\mathrm{n}=462)$, moderate anemia 90-95/L $(\mathrm{n}=88)$, severe anemia $<90 \mathrm{~g} / \mathrm{L}(\mathrm{n}=79)$

prior to infusion (Table 1). No differences were observed in hematological characteristics (hemoglobin and ferritin) at the booking appointment conducted at 12 week gestation (Table 2).

Following the infusion, samples were collected as required by the obstetric team for determination of hemoglobin values; data were available for $84 \%$ of the women at visit 1 (3 week post-infusion) and $34 \%$ of the women at visit 2 (6 week post-infusion). All women had at least one control visit. Changes in hemoglobin concentrations over the post-infusion period are presented in Fig. 1.

Hemoglobin levels collected at the first post-infusion time point ( 3 weeks) were significantly higher in all severity groups compared to pre-infusion levels (0 weeks) $(p<0.001$ for all severity groups). At the first post-infusion time point (3 weeks), hemoglobin had significantly increased by an average $( \pm$ SEM) of $5.1( \pm 1.3) \mathrm{g} / \mathrm{L}$ in women with ID and no anemia, $9.8( \pm 0.6) \mathrm{g} / \mathrm{L}$ in women with ID mild anemia, $15.3( \pm 1.2) \mathrm{g} / \mathrm{L}$ in women with ID moderate anemia, and $21.5( \pm 1.5) \mathrm{g} / \mathrm{L}$ in women with ID severe anemia. At 3 week post-infusion, hemoglobin concentrations were above the accepted adequate level for pregnant women $(110 \mathrm{~g} / \mathrm{L})$ in only the ID no anemia and the ID mild anemia groups (Fig. 1).

Hemoglobin concentrations did not significantly change between the first and second post-infusion visits (at 3 and
6 weeks) in both women with ID no anemia, and in women with ID and severe anemia (Fig. 1). However, in women with ID and both mild and moderate anemia, hemoglobin was significantly higher at the second compared to the first post-infusion visit ( $p<0.01$ for both). In women with mild anemia, the average ( \pm SEM) increase between 3 and 6 week post-infusion was $6.8(1.2) \mathrm{g} / \mathrm{L}$, while, in the moderate anemia group, the increase was $14.2(4.1) \mathrm{g} / \mathrm{L}$. At 6 week postinfusion, hemoglobin concentrations were above the recommended adequate level for pregnant women $(110 \mathrm{~g} / \mathrm{L})$ in all women except the ID severe anemia group (Fig. 1).

Ferritin values were available for a subset of women at each time visit and in the post-partum period. Ferritin significantly decreased between the booking and infusion visits $(p<0.001$; Table 3$)$. Ferritin levels at 3 week post-infusion were significantly higher than pre-infusion levels $(p<0.001)$ and remained elevated at 6 week post-infusion in the subset of women assessed $(n=28)$. In the subset of women who had ferritin measured post-partum $(n=27)$, levels were very low (Table 3).

Adverse reactions are presented in Table 4. Minor adverse events occurred in $96(11 \%)$ of women overall, with only $31(3.5 \%)$ women reporting more than one adverse effect. The most common adverse event was local irritation/pain of the skin at the site of infusion, which occurred in 29 women (3\%), while each of the other adverse effects was observed in less than $3 \%$ of women (Table 4). The frequency of adverse events differed significantly between the severity groups ( $p=0.029$ ), with the lowest number of adverse events observed in the no anemia group $(n=16,7 \%)$ and the highest rates observed in the mild $(n=54,12 \%)$ and moderate anemia groups $(n=18,20 \%)$. Fetal heart rate monitoring did not indicate a drug-related adverse effect on the fetal heart rate pattern.

Neonatal characteristics of the study population are presented in Table 5. Infants born to mothers with ID and moderate anemia had a significantly greater birth weight than those born to women without anemia $(p<0.01)$. All other neonatal anthropomorphic characteristics of the anemia groups did not differ significantly from each other. Apgar scores were similar across all anemia severities.
Table 3 Ferritin levels $(\mu \mathrm{g} / \mathrm{L})$ across the testing period

\begin{tabular}{llllll}
\hline & Booking & Pre-infusion & 3 week post-infusion & 6 week post-infusion & Post-partum \\
\hline Ferritin $(\mu \mathrm{g} / \mathrm{L})$ & $16(8-34)$ & $7(5-10)$ & $188(111-284)$ & $137(46-256)$ & $17(11-23)$ \\
& $n=514$ & $n=725$ & $n=106$ & $n=28$ & $n=27$ \\
\hline
\end{tabular}

Data are presented as median (IQR) 
Table 4 Adverse events in all women combined and according to severity of anemia

\begin{tabular}{|c|c|c|c|c|c|}
\hline Adverse events & All women $(n=863)$ & $\begin{array}{l}\text { ID no } \\
\text { anemia } \\
(n=234)\end{array}$ & $\begin{array}{l}\text { ID mild } \\
\text { anemia } \\
(n=462)\end{array}$ & $\begin{array}{l}\text { ID moder- } \\
\text { ate anemia } \\
(n=88)\end{array}$ & $\begin{array}{l}\text { ID severe } \\
\text { anemia } \\
(n=79)\end{array}$ \\
\hline Local (irritation/pain) & $36(4 \%)$ & $4(2 \%)$ & $26(6 \%)$ & $33(\%)$ & $3(4 \%)$ \\
\hline Headache/dizziness & $29(3 \%)$ & $6(3 \%)$ & $12(3 \%)$ & $10(11 \%)$ & $1(1 \%)$ \\
\hline Vascular (hypotension) & $26(3 \%)$ & $5(2 \%)$ & $14(3 \%)$ & $5(6 \%)$ & $2(2.5 \%)$ \\
\hline Nausea/vomiting & $21(2 \%)$ & $4(2 \%)$ & $11(2 \%)$ & $5(6 \%)$ & $1(1 \%)$ \\
\hline Musculoskeletal & $6(1 \%)$ & $0(0 \%)$ & $4(1 \%)$ & $2(2 \%)$ & $0(0 \%)$ \\
\hline Respiratory & $9(1 \%)$ & $2(1 \%)$ & $4(1 \%)$ & $1(1 \%)$ & $2(2.5 \%)$ \\
\hline $\begin{array}{l}\text { Total number of } \\
\text { patients reporting any } \\
\text { adverse event }\end{array}$ & $96(11 \%)$ & $16(7 \%)$ & $54(12 \%)$ & $18(20 \%)$ & $8(10 \%)$ \\
\hline $\begin{array}{l}\text { Patients reporting more } \\
\text { than one adverse } \\
\text { event }\end{array}$ & $31(3.5 \%)$ & $5(2 \%)$ & $17(4 \%)$ & $8(9 \%)$ & $1(1 \%)$ \\
\hline
\end{tabular}

Data are presented as $n$ (\% of women in same group)

Table 5 Neonatal characteristics of the whole cohort $(n=863)$ and according to anemia status at infusion

\begin{tabular}{|c|c|c|c|c|c|c|}
\hline & All births $(n=863)$ & ID no anemia $(n=234)$ & $\begin{array}{l}\text { ID mild anemia } \\
(n=462)\end{array}$ & $\begin{array}{l}\text { ID moderate } \\
\text { anemia }(n=88)\end{array}$ & $\begin{array}{l}\text { ID severe ane- } \\
\text { mia }(n=79)\end{array}$ & $p^{\mathrm{a}}$ \\
\hline Birth weight (g) & $3454 \pm 545$ & $3432 \pm 536$ & $3468 \pm 496$ & $3581 \pm 596^{\mathrm{b}}$ & $3308 \pm 726$ & 0.025 \\
\hline Length $(\mathrm{cm})$ & $50 \pm 2$ & $50 \pm 2$ & $50 \pm 2$ & $50 \pm 2$ & $49 \pm 3$ & 0.146 \\
\hline Head circumference $(\mathrm{cm})$ & $35 \pm 2$ & $35 \pm 2$ & $35 \pm 2$ & $35 \pm 2$ & $35 \pm 2$ & 0.672 \\
\hline Apgar 1 min & $9(8-9)$ & $9(8-9)$ & $9(8-9)$ & $9(8-9)$ & $9(8-9)$ & 0.324 \\
\hline Apgar $<7$ & $62(7 \%)$ & $15(6 \%)$ & $34(7 \%)$ & $8(9 \%)$ & $5(6 \%)$ & 0.895 \\
\hline Apgar 5 min & $9(9-9)$ & $9(9-9)$ & $9(9-9)$ & $9(9-9)$ & $9(9-9)$ & 0.379 \\
\hline Apgar $<7$ & $8(1 \%)$ & $3(1 \%)$ & $4(1 \%)$ & $1(1 \%)$ & $0(0 \%)$ & 0.345 \\
\hline
\end{tabular}

Data presented as mean $\pm \mathrm{SD}$, median (IQR) or $n(\%)$

${ }^{\mathrm{a}}$ Main effect of anemia severity groups

${ }^{\mathrm{b}} p<0.01$ versus no anemia (post hoc analysis)

\section{Discussion}

This large case series of 863 pregnant women strengthens the evidence on the safety and efficacy of IV iron administration with FCM in pregnancy in women. This includes 234 women who were iron-deficient and not anemic. No serious adverse events occurred amongst our patients, with only $11 \%$ of women experiencing mild adverse events, which were mostly self-limiting.

ID or IDA are very common conditions, with estimates that over $40 \%$ of women globally are affected [24]. ID and IDA increase maternal mortality and morbidity, particularly in the context of peri-partum hemorrhage, with rates of preterm delivery and low birth weight also increased [3]. While oral iron supplementation can increase hemoglobin and ferritin levels in pregnancy, we have previously demonstrated the safe and effective use of FCM infusion in pregnant women during the second and third trimesters of pregnancy [21]. Importantly, we also highlighted improved quality of life in these women, which has ongoing benefits to mental health and adoption of health-promoting behaviors [25]. We now expand this work to assess safety and efficacy of a single FCM infusion in women with differing severities of IDA and IDNA. This large cohort study demonstrates effective restoration of hemoglobin levels for up to 6 week post-infusion, regardless of anemia severity, with no serious adverse outcomes noted. The rate of minor adverse events was like that reported previously and was not amplified in any of the groups studied this time.

IDA has been the third leading cause globally for years lived with disability (YLDs) since 1990 and remained in this position over the last 2 decades [26]. In addition, it is ranked 13 for disability-adjusted life-years (DALYs) [27]. The impact on mental health, cognitive function, physical performance, work capacity, and general health is a substantial burden for the affected individual and society [9, 28, 29]. Personal and economic loss is enormous but preventable 
and treatable [30]. Progression to IDA poses additional risks and increases women's vulnerability; particularly should a peri-partum hemorrhage occur. Transfusion of blood products in the obstetric setting is not a rare event, varying between 1.6 and 3\% [12]. In addition, the incidence of peri-partum hemorrhage is on the rise and has increased by $33 \%$ between 2001 and 2010 [12]. Worldwide bleeding at childbirth remains the most common cause for maternal death [31]. Correcting hemoglobin by RBC transfusion remains a temporary measure if the underlying condition is not treated [32]. Optimizing iron stores prior to elective surgery associated with an increased risk of bleeding has been part of the wider concept of Patient Blood Management (PBM) and has been recommended in a consensus statement by international experts [33]. Women booked for elective caesarean section or entering labor with an increased risk of bleeding fall into the same category and deserve, therefore, to be managed accordingly $[16,17,34]$.

The significance and risk of ID for the child is well described. By treating ID in pregnancy, birthweight improves, and short and long-term consequences of ID can be prevented [3, 4, 35]. In the current cohort, the average birth weight was comparable to population norms (mean $3355 \mathrm{~g}$ ) [36]. Birth weight was not different between infants whose mothers had mild, moderate, and severe anemia, however, which was higher in the moderate anemia group compared to women with no anemia. While this was observed and the magnitude birthweight difference within a healthy weight range (approximately $150 \mathrm{~g}$ ) is clinically definitely significant, it is unlikely to be a direct consequence of FCM infusion, given that all women in the current study received this treatment, with no difference observed between the iron deficiency no anemia group compared to both the mild and severe groups.

The cycle from persistent ID in pregnancy to residual post-partum ID occurs in many women and can be compounded by heavy menstrual bleeding [25]. Fatigue is one of the most common presentations in the post-partum period, associated with low concentration, low mood, depressive symptoms, and irritability [29]. All will impact on ability to care for the newborn [37-39]. This raises the question for the ideal treatment approach for women with iron deficiency and emphasizes the importance of follow-up and potential ongoing treatment in the post-partum period. Interestingly, six women from our previous publication [21] were in this new cohort in their consecutive pregnancy where they again presented with profound ID or IDA. Despite intravenous iron replacement therapy in their previous pregnancy, these women were unable to sustain normal iron stores into and during their next pregnancy. Addressing ID in pregnancy and beyond, therefore, becomes a crucial component of antenatal and postnatal care and appears to gain wider acceptance amongst clinicians [22].

A limitation of our study is the lack of a control group and the retrospective nature of the data. The strength, however, is that this large cohort reflects clinical practice of a large obstetric unit at a university teaching hospital in metropolitan setting, and shows clear benefit of this therapy in improving iron stores in women with ID.

In conclusion, our results strengthen the evidence that IV iron in the form of FCM is safe and efficacious to treat IDA and IDNA in pregnancy. The timely detection and appropriate treatment of ID can significantly improve maternal health and pregnancy outcomes and may offer a circuit breaker for women with one of the most common diseases globally.

Acknowledgements We would like to thank all midwifes in Women's Assessment Unit of the Lyell McEwin Hospital, who collected the adverse event information during the infusion. A special thank you to Tanya Warne (Midwife), Emily Bliss (Medical Student) and Natalie Aboustate (Research Assistant) for their help with case note review and data entry.

Author contributions BF: protocol/project development, data collection, data analysis, and manuscript writing. TG: data collection. GD: protocol/project development and manuscript editing. NAH: protocol/ project development, data analysis, and manuscript editing. All authors read and approved the final manuscript.

\section{Compliance with ethical standards}

Conflict of interest BF has received financial support to give lectures, undertake research, attend scientific advisory boards, and undertake consultancies for the New South Wales Department of Health, South Australia Department of Health, Australian Red Cross Blood Service, Australian National Blood Authority, Vifor Pharma Ltd., Switzerland, and CSL Behring Australia. No support was received from any organization for the submitted work. TG, GD, and NH declare that they have no conflict of interest.

Open Access This article is distributed under the terms of the Creative Commons Attribution 4.0 International License (http://creativeco mmons.org/licenses/by/4.0/), which permits unrestricted use, distribution, and reproduction in any medium, provided you give appropriate credit to the original author(s) and the source, provide a link to the Creative Commons license, and indicate if changes were made.

\section{References}

1. Bencaiova G, Burkhardt T, Breymann C (2012) Anemiaprevalence and risk factors in pregnancy. Eur J Intern Med 23(6):529-533. https://doi.org/10.1016/j.ejim.2012.04.008

2. Khalafallah AA, Dennis AE, Ogden K et al (2012) Three-year follow-up of a randomised clinical trial of intravenous versus oral iron for anaemia in pregnancy. BMJ Open. https://doi. org/10.1136/bmjopen-2012-000998

3. Haider BA, Olofin I, Wang M et al (2013) Anaemia, prenatal iron use, and risk of adverse pregnancy outcomes: 
systematic review and meta-analysis. BMJ 346:f3443. https:// doi.org/10.1136/bmj.f3443

4. Congdon EL, Westerlund A, Algarin CR et al (2012) Iron deficiency in infancy is associated with altered neural correlates of recognition memory at 10 years. J Pediatr 160(6):1027-1033. https://doi.org/10.1016/j.jpeds.2011.12.011

5. Pratt JJ, Khan KS (2015) Non-anaemic iron deficiency-a disease looking for recognition of diagnosis: a systematic review. Eur J Haematol. https://doi.org/10.1111/ejh.12645

6. Azulay CE, Pariente G, Shoham-Vardi I et al (2015) Maternal anemia during pregnancy and subsequent risk for cardiovascular disease. J Matern Fetal Neonatal Med 28(15):1762-1765. https ://doi.org/10.3109/14767058.2014.971743

7. Favrat B, Balck K, Breymann C et al (2014) Evaluation of a single dose of ferric carboxymaltose in fatigued, iron-deficient women-PREFER a randomized, placebo-controlled study. PLoS One 9(4):e94217. https://doi.org/10.1371/journ al.pone. 0094217

8. Miles LF, Kunz SA, Na LH et al (2017) Postoperative outcomes following cardiac surgery in non-anaemic iron-replete and irondeficient patients—an exploratory study. Anaesthesia. https://doi. org/10.1111/anae.14115

9. Scott SP, Murray-Kolb LE (2016) Iron status is associated with performance on executive functioning tasks in nonanemic young women. J Nutr 146(1):30-37. https://doi.org/10.3945/ jn. 115.223586

10. Abir G, Mhyre J (2017) Maternal mortality and the role of the obstetric anesthesiologist. Best Pract Res Clin Anaesthesiol 31(1):91-105. https://doi.org/10.1016/j.bpa.2017.01.005

11. Roberts CL, Nippita TA (2015) International caesarean section rates: the rising tide. Lancet Glob Health 3(5):e241-e242. https ://doi.org/10.1016/s2214-109x(15)70111-7

12. Patterson JA, Roberts CL, Bowen JR et al (2014) Blood transfusion during pregnancy, birth, and the postnatal period. Obstet Gynecol 123(1):126-133. https://doi.org/10.1097/aog.00000 00000000054

13. Goodnough LT (2013) Blood management: transfusion medicine comes of age. Lancet 381(9880):1791-1792. https://doi. org/10.1016/S0140-6736(13)60673-X

14. Kickler TS, Smith B, Bell W et al (1985) Estimation of transfused red cell survival using an enzyme-linked antiglobulin test. Transfusion 25(5):401-405. https://doi.org/10.104 6/j.1537-2995.1985.25586020109.x

15. Froessler B, Palm P, Weber I et al (2016) The important role for intravenous iron in perioperative patient blood management in major abdominal surgery: a randomized controlled trial. Ann Surg 264(1):41-46. https://doi.org/10.1097/sla.0000000000001646

16. Flores CJ, Sethna F, Stephens B et al (2017) Improving patient blood management in obstetrics: snapshots of a practice improvement partnership. BMJ Qual Improv Rep. https://doi.org/10.1136/ bmjquality-2017-000009

17. Breymann C, Honegger C, Hosli I et al (2017) Diagnosis and treatment of iron-deficiency anaemia in pregnancy and postpartum. Arch Gynecol Obstet. https://doi.org/10.1007/s00404-017-4526-2

18. Froessler B, Dekker G, McAuliffe G (2015) To the rescue: the role of intravenous iron in the management of severe anaemia in the peri-partum setting. Blood Transfus (Trasfusione del sangue) 13(1):150-152. https://doi.org/10.2450/2014.0220-14

19. Ivana Chandra L-zS (2015) Iron status and choice of iron therapy during pregnancy: advantages and disadvantages. Int J Reprod Contracept Obstet Gynecol 4(5):1264-1271

20. Mayson E, Ampt AJ, Shand AW et al (2015) Intravenous iron: barriers and facilitators to its use at nine maternity hospitals in New South Wales, Australia. Aust N Z J Obstet Gynaecol. https ://doi.org/10.1111/ajo.12417
21. Froessler B, Collingwood J, Hodyl NA et al (2014) Intravenous ferric carboxymaltose for anaemia in pregnancy. BMC Pregnancy Childbirth 14:115. https://doi.org/10.1186/1471-2393-14-115

22. Daru J, Moores R, Dodds J et al (2015) Non-anaemic iron deficiency in pregnancy: the views of health service users and health care professionals. Transfus Med 25(1):27-32. https://doi. org/10.1111/tme.12184

23. Christoph P, Schuller C, Studer H et al (2012) Intravenous iron treatment in pregnancy: comparison of high-dose ferric carboxymaltose vs. iron sucrose. J Perinat Med 40(5):469-474. https:// doi.org/10.1515/jpm-2011-0231

24. Sweet MG, Schmidt-Dalton TA, Weiss PM et al (2012) Evaluation and management of abnormal uterine bleeding in premenopausal women. Am Fam Physician 85(1):35-43

25. Friedman AJ, Chen Z, Ford P et al (2012) Iron deficiency anemia in women across the life span. J Womens Health (Larchmt) 21(12):1282-1289. https://doi.org/10.1089/jwh.2012.3713

26. Vos T, Flaxman AD, Naghavi M et al (2012) Years lived with disability (YLDs) for 1160 sequelae of 289 diseases and injuries 1990-2010: a systematic analysis for the Global Burden of Disease Study 2010. Lancet 380(9859):2163-2196. https://doi. org/10.1016/S0140-6736(12)61729-2

27. Murray CJL, Lopez AD (2013) Measuring the global burden of disease. N Engl J Med 369(5):448-457. https://doi.org/10.1056/ NEJMra1201534

28. Kassebaum NJ, Bertozzi-Villa A, Coggeshall MS et al (2014) Global, regional, and national levels and causes of maternal mortality during 1990-2013: a systematic analysis for the Global Burden of Disease Study 2013. Lancet 384(9947):980-1004. https:// doi.org/10.1016/s0140-6736(14)60696-6

29. Albacar G, Sans T, Martin-Santos R et al (2011) An association between plasma ferritin concentrations measured $48 \mathrm{~h}$ after delivery and postpartum depression. J Affect Disord 131(1-3):136142. https://doi.org/10.1016/j.jad.2010.11.006

30. WHO (2013) Micronutrient deficiencies. http://www.who.int/nutri tion/topics/ida/en/. Accessed 29 Apr 2016

31. Collis RE, Collins PW (2015) Haemostatic management of obstetric haemorrhage. Anaesthesia 70(Suppl 1):78-86. https://doi. org/10.1111/anae.12913 (e27-e78)

32. Froessler B, Palm P, Weber I et al (2016) The important role for intravenous iron in perioperative patient blood management in major abdominal surgery: a randomized controlled trial. Ann Surg. https://doi.org/10.1097/sla.0000000000001646

33. Munoz M, Acheson AG, Auerbach M et al (2016) International consensus statement on the peri-operative management of anaemia and iron deficiency. Anaesthesia. https://doi.org/10.1111/ anae. 13773

34. Froessler B, Mol B, Dekker G et al (2017) Anaemic parturient and the anaesthesiologist: are we asleep at the wheel? Eur J Anaesthesiol 34(7):405-407. https://doi.org/10.1097/eja.000000000000060 0

35. McArdle HJ, Gambling L, Kennedy C (2014) Iron deficiency during pregnancy: the consequences for placental function and fetal outcome. Proc Nutr Soc 73(1):9-15. https://doi.org/10.1017/s0029 665113003637

36. Australian Institute of Health and Welfare (2015) Australia's mothers and babies 2013 -in brief. Perinatal statistics series no. 31. Cat no. PER 72. AIHW, Canberra

37. Breymann C, Gliga F, Bejenariu C et al (2008) Comparative efficacy and safety of intravenous ferric carboxymaltose in the treatment of postpartum iron deficiency anemia. Int J Gynaecol Obstet 101(1):67-73

38. Breymann C, Milman N, Mezzacasa A et al (2016) Ferric carboxymaltose vs. oral iron in the treatment of pregnant women with iron deficiency anemia: an international, open-label, 
randomized controlled trial (FER-ASAP). J Perinat Med. https:// doi.org/10.1515/jpm-2016-0050

39. Markova V, Norgaard A, Jorgensen KJ et al (2015) Treatment for women with postpartum iron deficiency anaemia. Cochrane
Database Syst Rev 8:Cd010861. https://doi.org/10.1002/14651 858.cd010861.pub2 